\title{
EXTENSION OF VECTOR-LATTICE HOMOMORPHISMS
}

\section{Z. LIPECKI}

ABSTRACT. Extensions of a positive linear operator on a vector lattice satisfying a certain approximation condition are considered. When the operator is a vectorlattice homomorphism, these extensions are also vector-lattice homomorphisms.

We shall give a short and direct proof of the following result which can also be derived by combining Theorem 3 of [1] and Theorem 1 of [2].

THEOREM. Let $X$ and $Y$ be vector lattices over the reals and let $Y$ be order complete. Suppose $M$ is a cofinal vector subspace of $X$ and $T: M \rightarrow Y$ is a positive linear operator. Then $T$ extends to a positive linear operator $S: X \rightarrow Y$ with the property inf $\{S(|x-z|): z \in M\}=0$ for each $x \in X$.

Proof. Denote by $\mathbf{M}$ the class of all pairs $(N, P)$, where $N$ is a vector subspace of $X$ with $M \subset N$ and $P: N \rightarrow Y$ is a positive linear operator such that $P \mid M=T$ and $\inf \left\{P_{e}(|v-z|): z \in M\right\}=0$ for each $v \in N$. Here $P_{e}$ denotes the "outer" operator generated by $P$, i.e., $P_{e}(x)=\inf \{P(v): x<v \in N\}$ for $x \in X$. We define an ordering $\ll$ in $\mathbf{M}$ by putting $\left(N_{1}, P_{1}\right) \ll\left(N_{2}, P_{2}\right)$ whenever $N_{1} \subset N_{2}$ and $P_{2} \mid N_{1}=P_{1}$.

Step I. If $(N, P) \in \mathbf{M}$ and $x_{0} \in X$, then there exists a positive linear operator $P_{0}$ on the linear subspace $N_{0}$ generated by $N \cup\left\{x_{0}\right\}$ such that $\left(N_{0}, P_{0}\right) \in M$ and $(N, P) \ll\left(N_{0}, P_{0}\right)$. Indeed, put $P_{0}\left(v+t x_{0}\right)=P(v)+t P_{e}\left(x_{0}\right)$ for $v \in N$ and $t \in$ $R$. Clearly, $P_{0}$ is linear and $P_{0} \mid N=P$. In order to show that $P_{0}$ is positive assume $v+t x_{0}>0$. If $t>0$, we have $x_{0} \geqslant-v / t$ which yields $P_{e}\left(x_{0}\right)>-P(v) / t$. Hence $P_{0}\left(v+t x_{0}\right) \geqslant 0$. Analogously, the same holds if $t<0$. By the sublinearity of $P_{0 e}$, the set of all $x \in X$ with the property $\inf \left\{P_{0 e}(|x-v|): v \in N\right\}=0$ is a linear subspace of $X$. As, by definition, $\inf \left\{P_{0}\left(v-x_{0}\right): x_{0}<v \in N\right\}=0$, this subspace contains $N_{0}$. Moreover, we have

$$
\begin{aligned}
P_{0 e}(|x-z|) & \leqslant P_{0 e}(|x-v|)+P_{0 e}(|v-z|) \\
& \leqslant P_{0 e}(|x-v|)+P_{e}(|v-z|) .
\end{aligned}
$$

It follows that $\left(N_{0}, P_{0}\right) \in \mathbf{M}$.

Step II. According to the Kuratowski-Zorn lemma, there exists a maximal element $(N, S)$ of $\mathbf{M}$ with respect to $\ll$. By Step I, we have $N=X$ which proves the theorem.

Received by the editors October 10, 1978 and, in revised form, April 10, 1979.

AMS (MOS) subject classifications (1970). Primary 47B55; Secondary 46A40.

Key words and phrases. Vector lattice, positive operator, vector-lattice homomorphism. 
As proved in [1], the approximation condition appearing above characterizes the extreme extensions of a positive linear operator. In this connection let us note the following simple consequence of the theorem.

COROLlary ([2, Corollary 2]). Let $X$ and $Y$ be as in the theorem. Suppose $M$ is a cofinal vector sublattice of $X$ and $T: M \rightarrow Y$ is a vector-lattice homomorphism. Then $T$ extends to a vector-lattice homomorphism $S: X \rightarrow Y$.

Finally, let us observe that both the theorem and its corollary fail for latticeordered abelian groups.

EXAMPLE. Let $X$ and $Y$ be the integers with the usual addition and ordering and choose $M$ to be the even integers. Then $T: M \rightarrow Y$ defined by $T(2 n)=n$ is an additive lattice homomorphism which has no additive extension to the whole of $X$.

ADDED IN PROOF. 1. The corollary has been also proved, independently and by still another method, by W. A. J. Luxemburg and A. R. Schep (An extension theorem for Riesz homomorphisms, Indag. Math. 41 (1979), 145-154; Theorem 3.1).

2. Both the theorem and its corollary can be generalized to the case where $X$ is a directed ordered vector space and $M$ is a directed vector subspace of $X$. In this setting " $S(|x-z|)$ " is replaced by " $S_{m}(x-z)$ ", where $S_{m}(x)=\inf \{S(v): \pm x<v$ $\in X\}$ (see Z. Lipecki, Extensions of positive operators and extreme points. III, Colloq. Math. (to appear)). The proof presented here can also be easily adapted to this general case.

\section{REFERENCES}

1. Z. Lipecki, D. Plachky and W. Thomsen, Extensions of positive operators and extreme points. I, Colloq. Math. 42 (to appear).

2. Z. Lipecki, Extensions of positive operators and extreme points. II, Colloq. Math. 42 (to appear).

Institute of Mathematics, Polish Academy of Sciences, Kopernika 18, 51-617 Wrockaw, Poland 